\title{
Transcriptional induction of IRF7 and IRF9 in coronavirus infections.
}

Shahan Mamoor, MS ${ }^{1}$

1shahanmamoor@gmail.com

East Islip, NY USA

The host transcriptional response provides critical insights into host-pathogen interactions. We mined multiple independent published microarray datasets ${ }^{1-5}$ and discovered transcriptional induction of the interferon regulatory factors IRF7 and IRF9 in vitro following infection of human cells with an isolate of the Middle East Respiratory Syndrome coronavirus (MERS-CoV) and in vivo in the lungs of mice following infection with Severe Acute Respiratory Syndrome coronavirus 1 (SARS-CoV-1), but not after infection of human cells with human coronavirus 229E (HCoV 229E). These data reveal a conserved interferon transcriptional response among MERS- and SARS-CoV and suggest that interferon signaling downstream of IRF7 and IRF9 might be important events during coronavirus infections.

Keywords: coronavirus, SARS-CoV-1, MERS-CoV, HCOV-229E, systems biology of viral infections, IRF7, IRF9, interferon signaling. 
The novel coronavirus SARS-CoV-2 has infected close to $20,000,000$ people worldwide in less than one year6. We mined published microarray data to understand the host transcriptional response to coronavirus infection: in vitro, following infection with MERS-CoV and HCoV-229E infection and in vivo following infection with SARSCoV-1 in the lungs of mice and ferrets. We discovered a conserved interferon transcriptional response among specific members of the coronavirus family involving transcriptional induction of the transcription factors and interferon regulatory factors IRF7 and IRF9.

\section{Methods}

We used datasets GSE56677², GSE59185², GSE688204, GSE225815 and GSE891676 for this analysis of gene expression changes following coronavirus infection. GSE56677 was generated using Agilent-039494 SurePrint G3 Human GE v2 8x60K Microarray 039381 technology with $n=3$ each for CALU3 2B4 cells infected with MERS-CoV London, at 0 hours, 3 hours, 7 hours, 12 hours, 18 hours and 24 hours post-infection. GSE59185 was generated using Agilent-028005 SurePrint G3 Mouse GE 8x60K Microarray technology with $n=3$ mock-infected lung and $n=3$ SARS-CoV-1infected lung at 2 days post-infection in BALB/c mice. GSE68820 was generated using Agilent-014868 Whole Mouse Genome Microarray 4x44K G4122F technology with $n=5$ for lung from mock-infected C57BL6/NJ mice at 2 days post-infection and $n=5$ for lung from SARS-CoV-1-infected mice at 2 days post-infection. GSE22581 was generated using Affymetrix Canine Genome 2.0 Array technology with $n=3$ each for ferret lung on day 0, day 1 and day 2 post-infection with SARS-CoV-1. GSE89167 was generated 
using 039494 SurePrint G3 Human GE v2 8x60K Microarray 039381 technology with $n=2 \mathrm{HuH}-7$ cells and $n=2 \mathrm{HuH}-7$ cells infected with HCoV229E.

The Benjamin and Hochberg method of $p$-value adjustment was used for ranking of differential expression but raw p-values were used for assessment of statistical significance of global differential expression. Log-transformation of data was autodetected, and the NCBI generated category of platform annotation was used. GEO2R only provides exact mRNA expression levels for the 250 most differentially expressed genes and thus fold change is calculated only for transcripts ranking 1-250.

A statistical test was performed to evaluate the significance of difference in IRF9 mRNA expression levels in CALU3 2B4 cells with MERS-CoV infection as compared to CALU3 2B4 cells at baseline (0 hours) using a one-way ANOVA with Dunnett's multiple comparisons test. A statistical test was performed to evaluate the significance of difference in IRF7 mRNA expression levels in the lungs of BALB/c mice and in the lungs of C57BL6/NJ mice at 2 days post-infection with SARS-CoV-1 using a two-tailed, unpaired t-test with Welch's correction. We used PRISM for all statistical analyses (Version 8.4.0)(455).

\section{Results}

We mined multiple published microarray datasets to determine in an unbiased and systematic fashion the genes most differentially expressed following infection of cells and tissues with multiple members of the coronavirus family.

IRF9 and IRF7 are differentially expressed following infection of human cells with MERS-CoV London. 
In CALU3 2B4 cells infected with MERS-CoV London (Eng1), an isolate of MERS-CoV, we observed significant differential expression of the transcription factor and interferon regulatory factor IRF9. When sorting each of the transcripts expressed in CALU3 cells following infection with MERS-CoV London based on significance of difference in expression between uninfected and infected cells, IRF9 ranked 189 out of 28653 total transcripts (Table 1). Differential expression of IRF9 in CALU3 cells following MERS-CoV infection was statistically significant (Table $1 ; p=8.21 \mathrm{E}-12$ ).

A second interferon regulatory factor, IRF7 was also among the genes most differentially expressed in CALU3 cells following infection with MERS-CoV London. When sorting each of the transcripts expressed in CALU3 cells following infection with MERS-CoV London based on significance of difference in expression between uninfected and infected cells, IRF7 ranked 577 out of 28653 total transcripts (Table 1). Differential expression of IRF7 in CALU3 cells following MERS-CoV infection was statistically significant (Table $1 ; p=3.77 \mathrm{E}-10$ ).

IRF9 and IRF7 are differentially expressed in the mouse lung following infection with SARS-CoV-1.

We referenced two separate microarray datasets, generated using SARS-CoV-1 infection of BALB/c mice and of C57BL6/NJ mice, to determine if differential expression of interferon regulatory factors IRF7 and IRF9 was conserved in mice and across coronavirus type.

Again, we found that IRF7 and IRF9 were among the genes most differentially expressed in the lungs of mice infected with another human coronavirus, SARS-CoV-1 
(Table 2). When sorting each of the transcripts expressed in the lungs of BALB/c mice following infection with SARS-CoV-1 based on significance of difference in expression between mock-infected and infected mice, IRF9 ranked 1088 out of 62976 total transcripts (Table 2). Differential expression of IRF9 in the mouse lung following SARSCoV-1 infection was statistically significant (Table 2; $p=1.2 \mathrm{E}-05$ ). When sorting each of the transcripts expressed in the lungs of BALB/c mice following infection with SARSCoV-1 based on significance of difference in expression between mock-infected and infected mice, IRF7 ranked 85 out of 62976 total transcripts (Table 2). Differential expression of IRF7 in the mouse lung following SARS-CoV-1 infection was statistically significant (Table 2; $p=1.51 \mathrm{E}-08$ ).

We observed a similar transcriptional response to SARS-CoV-1 infection in C57BL6/NJ mice (Table 3). When sorting each of the transcripts expressed in the lungs of C57BL6/NJ mice following infection with SARS-CoV-1 based on significance of difference in expression between mock-infected and infected mice, IRF9 ranked 747 out of 29649 total transcripts (Table 2). Differential expression of IRF9 in the mouse lung following SARS-CoV-1 infection was statistically significant (Table $3 ; p=1.6 \mathrm{E}-09$ ). When sorting each of the transcripts expressed in the lungs of C57BL6/NJ mice following infection with SARS-CoV-1 based on significance of difference in expression between mock-infected and infected mice, IRF7 ranked 4 out of 29649 total transcripts (Table 3). Differential expression of IRF7 in the mouse lung following SARS-CoV-1 infection was statistically significant (Table $3 ; p=3.36 \mathrm{E}-15$ ). 
IRF9 but not IRF7 is differentially expressed in the ferret lung following SARS-CoV-1 infection.

Next, we referenced a microarray dataset generated from the lungs and blood of ferrets infected through the intranasal route with SARS-CoV-1 to determine if differential expression of IRF7 and IRF9 following a coronavirus infection could be observed in a separate and important small animal model of coronavirus infection. IRF9 but not IRF7 was differentially expressed in the ferret lung following intranasal infection with SARS-CoV-1; this was less marked than observed in vitro with MERSCoV infection and in mice with SARS-CoV-1 infection. When sorting each of the transcripts expressed in the ferret lung following infection with SARS-CoV-1 based on significance of difference in expression between mock-infected and infected ferrets, IRF9 ranked 23168 out of 43035 total transcripts (Table 4). Differential expression of IRF9 in the ferret lung following SARS-CoV-1 infection was statistically significant (Table 4; $p=3.02 \mathrm{E}-01$ ), but IRF7 was not (Table 4; $p=2.14 \mathrm{E}-01$ ).

When sorting each of the transcripts expressed in the blood of ferrets following intranasal infection with SARS-CoV-1 based on significance of difference in expression between mock-infected and infected ferrets, IRF7 ranked 15588 out of 43035 total transcripts (Table 5). Differential expression of IRF7 in the blood of ferrets following SARS-CoV-1 infection was not statistically significant (Table $5 ; p=4.72 \mathrm{E}-01$ ), nor was differential expression of IRF9 in the ferret blood (Table 5; $p=4.72 E-01$ ). 
Finally, we asked whether infection with the human coronavirus $229 \mathrm{E}$ (HCoV-229E). Neither IRF7 nor IRF9 were differentially expressed in the Huh-7 human cell line following infection with HCoV-229E in vitro (Table 6).

Rapid transcriptional induction of IRF9 in vitro following MERS-CoV infection.

We assessed kinetics of IRF9 transcription following MERS-CoV London (Eng1) infection of CALU3 2B4 cells in vitro (Figure 1). By 12 hours post-infection, IRF9 mRNA levels increased and this change approached the level of statistical significance (Figure $1 ; p=0.0601$ ), with a mean fold increase of $1.049 \pm 0.031$ in IRF9 mRNA at 12 hours post-infection. At 18 and 24 hours post-infection, levels of IRF9 transcript in CALU3 cells were significantly induced as compared to baseline ( 0 hours) (Figure $1 ; p<0.0001$ at 18 hours and $p<0.0001$ at 24 hours, respectively), with mean fold induction of IRF9 mRNA of $1.239 \pm 0.011$ at 18 hours and $1.294 \pm 0.010$ at 24 hours (Table 1 ).

Transcriptional induction of IRF7 in the mouse lung in vivo following infection with SARS-CoV-1.

We also obtained exact mRNA expression levels of the IRF7 transcription factor following SARS-CoV-1 infection of the mouse lung in vivo (Figure 2 and Figure 3). At 2 days post-infection in BALB/c mice, IRF7 mRNA levels were significantly increased and this change was statistically significant (Figure 2; $p<0.0001$ ), with a mean fold increase of $1.67 \pm 0.04$ in IRF7 transcript levels. In C57BL6/NJ mice, at 2 days post-infection with SARS-CoV-1, IRF7 mRNA levels significantly increased in the lungs (Figure 3; $p<0.0001$ ), with a mean fold increase of IRF7 mRNA of $1.7104 \pm 0.0103$. 
Thus, by mining global transcriptome data from five separate coronavirus infection models in vitro and in vivo we uncovered significant differential expression and transcriptional induction of the transcription factors IRF7 and IRF9 as part of the host transcriptional response to SARS-CoV-1 and MERS-CoV London (Eng1) infection, but not in response to HCoV-229E infection. In vitro, IRF9 induction in response to coronavirus infection could be observed as early as 12 hours post-infection.

\section{Discussion}

Interferon regulatory factors, including IRF7 and IRF9 are transcription factors that can occupy interferon response elements to trans-activate interferon gene expression for anti-viral signaling. IRF7 has been described as the master regulator of type I interferon signaling in the immune system 7 . IRF7 can propagate signal transduction downstream of Toll-like receptors leading to interferon alpha activation through interactions with the cytoplasmic adapters TRAF6 and MyD888. Humans with genetic deficiency in IRF7 suffer from severe influenza infections and display impaired interferon signaling?

IRF9 is also important for anti-viral immune responses. An important binding partner of IRF9 in effecting immune defense is STAT2 ${ }^{10-12}$, which can cooperate with NF-kB to induce expression of the cytokine interleukin-6, binding IRF9 through a carboxy-terminal IRF association domain for induction of interferon stimulated genes (ISG). Interestingly, severe influenza infection has also been documented in a child with genetic deficiency in IRF913. A family with IRF9 deficiency has been described; fibroblasts from this family fail to induce expression of ISG in response to respiratory 
syncytial virus (RSV) infection ${ }^{14}$. Thus, studies from humans with genetic deficiencies in IRF7 and IRF9 demonstrate that these interferon regulatory transcription factors are essential for immune defense to viral infection of the respiratory tract.

We describe here the differential expression and transcriptional induction of transcription factors IRF7 and IRF9 in multiple coronavirus infection models: in MERSCoV infection of human cells in vitro and in the mouse lung following SARS-CoV-1 infection in two strains of mice in vivo. IRF7 and IRF9 may be important for SARSCoV-2 immune defense in humans. 


\section{References}

1. Selinger, C., Tisoncik-Go, J., Menachery, V.D., Agnihothram, S., Law, G.L., Chang, J., Kelly, S.M., Sova, P., Baric, R.S. and Katze, M.G., 2014. Cytokine systems approach demonstrates differences in innate and pro-inflammatory host responses between genetically distinct MERS-CoV isolates. BMC genomics, 15(1), p.1161.

2. Regla-Nava, J.A., Nieto-Torres, J.L., Jimenez-Guardeño, J.M., Fernandez-Delgado, R., Fett, C., Castaño-Rodríguez, C., Perlman, S., Enjuanes, L. and DeDiego, M.L., 2015. Severe acute respiratory syndrome coronaviruses with mutations in the $E$ protein are attenuated and promising vaccine candidates. Journal of virology, 89(7), pp.3870-3887

3. Totura, A.L., Whitmore, A., Agnihothram, S., Schäfer, A., Katze, M.G., Heise, M.T. and Baric, R.S., 2015. Toll-like receptor 3 signaling via TRIF contributes to a protective innate immune response to severe acute respiratory syndrome coronavirus infection. MBio, 6(3), pp.e00638-15.

4. Danesh, A., Cameron, C.M., León, A.J., Ran, L., Xu, L., Fang, Y., Kelvin, A.A., Rowe, T., Chen, H., Guan, Y. and Jonsson, C.B., 2011. Early gene expression events in ferrets in response to SARS coronavirus infection versus direct interferon-alpha2b stimulation. Virology, 409(1), pp.102-112.

5. Poppe, M., Wittig, S., Jurida, L., Bartkuhn, M., Wilhelm, J., Müller, H., Beuerlein, K., Karl, N., Bhuju, S., Ziebuhr, J. and Schmitz, M.L., 2017. The NF-kB-dependent andindependent transcriptome and chromatin landscapes of human coronavirus $229 \mathrm{E}-$ infected cells. PLoS pathogens, 13(3), p.e1006286.

6. World Health Organization. Coronavirus disease (COVID-19) pandemic. https:// www.who.int/emergencies/diseases/novel-coronavirus-2019.

7. Honda, K., Yanai, H., Negishi, H., Asagiri, M., Sato, M., Mizutani, T., Shimada, N., Ohba, Y., Takaoka, A., Yoshida, N. and Taniguchi, T., 2005. IRF-7 is the master regulator of type-I interferon-dependent immune responses. Nature, 434(7034), pp. 772-777.

8. Kawai, T., Sato, S., Ishii, K.J., Coban, C., Hemmi, H., Yamamoto, M., Terai, K., Matsuda, M., Inoue, J.I., Uematsu, S. and Takeuchi, O., 2004. Interferon-a induction through Toll-like receptors involves a direct interaction of IRF7 with MyD88 and TRAF6. Nature immunology, 5(10), pp.1061-1068. 
9. Ciancanelli, M.J., Huang, S.X., Luthra, P., Garner, H., Itan, Y., Volpi, S., Lafaille, F.G., Trouillet, C., Schmolke, M., Albrecht, R.A. and Israelsson, E., 2015. Life-threatening influenza and impaired interferon amplification in human IRF7 deficiency. Science, 348(6233), pp.448-453.

10.Kraus, T.A., Lau, J.F., Parisien, J.P. and Horvath, C.M., 2003. A hybrid IRF9-STAT2 protein recapitulates interferon-stimulated gene expression and antiviral response. Journal of Biological Chemistry, 278(15), pp.13033-13038.

11.Nan, J., Wang, Y., Yang, J. and Stark, G.R., 2018. IRF9 and unphosphorylated STAT2 cooperate with NF-KB to drive IL6 expression. Proceedings of the National Academy of Sciences, 115(15), pp.3906-3911.

12.Platanitis, E., Demiroz, D., Schneller, A., Fischer, K., Capelle, C., Hartl, M., Gossenreiter, T., Müller, M., Novatchkova, M. and Decker, T., 2019. A molecular switch from STAT2-IRF9 to ISGF3 underlies interferon-induced gene transcription. Nature communications, 10(1), pp.1-17.

13.Hernandez, N., Melki, I., Jing, H., Habib, T., Huang, S.S., Danielson, J., Kula, T., Drutman, S., Belkaya, S., Rattina, V. and Lorenzo-Diaz, L., 2018. Life-threatening influenza pneumonitis in a child with inherited IRF9 deficiency. The Journal of experimental medicine, 215(10), pp.2567-2585.

14.García-Morato, M.B., Apalategi, A.C., Bravo-Gallego, L.Y., Moreno, A.B., SimónFuentes, M., Garmendia, J.V., Echevarría, A.M., del Rosal Rabes, T., DomínguezSoto, Á., López-Granados, E. and Reyburn, H.T., 2019. Impaired control of multiple viral infections in a family with complete IRF9 deficiency. Journal of Allergy and Clinical Immunology, 144(1), pp.309-312. 

MERS-CoV London infection in vitro.

Rank of differential expression transcriptome-wide, probe ID, p-value, F statistic, fold change (FC) in mRNA expression level as compared to baseline, gene and gene name are listed in this chart. 


\begin{tabular}{|r|l|l|l|l|l|l|l|}
\hline \multicolumn{1}{|l|}{ Rank } & ID & p-value & t & B & FC & Gene & Gene name \\
\hline $\mathbf{8 5}$ & 36354 & $1.51 \mathrm{E}-08$ & $2.59 \mathrm{E}+01$ & 10.48361 & $1.67 \pm 0.04$ & Irf7 & $\begin{array}{l}\text { interferon } \\
\text { regulatory factor 7 }\end{array}$ \\
\hline $\mathbf{1 0 8 8}$ & 47484 & $1.2 \mathrm{E}-05$ & $1.03 \mathrm{E}+01$ & 3.79137 & & Irf9 & $\begin{array}{l}\text { interferon } \\
\text { regulatory factor 9 }\end{array}$ \\
\hline
\end{tabular}

Table 2: IRF7 and IRF9 are differentially expressed in the lung following infection with SARS-CoV-1 in BALB/c mice.

Rank of differential expression transcriptome-wide, probe ID, $p$-value, $t$, a moderated tstatistic, B, the log-odds of differential expression between the groups compared, fold change (FC) in mRNA expression level as compared to baseline, gene and gene name are listed in this chart. 


\begin{tabular}{|r|l|l|l|l|l|l|l|}
\hline \multicolumn{1}{|r}{ Rank } & ID & p-value & t & B & FC & Gene & Gene name \\
\hline $\mathbf{4}$ & A_51_P421876 & $3.36 \mathrm{E}-15$ & 69.57809 & 24.983928 & $1.7104 \pm$ & Irf7 & $\begin{array}{l}\text { interferon } \\
\text { regulatory factor 7 }\end{array}$ \\
\hline $\mathbf{7 4 7}$ & A_51_P127367 & $1.6 \mathrm{E}-0103$ & 19.52095 & 12.342548 & & Iff9 & $\begin{array}{l}\text { interferon } \\
\text { regulatory factor 9 }\end{array}$ \\
\hline
\end{tabular}

Table 3: IRF7 and IRF9 are differentially expressed in the lung following infection with SARS-CoV-1 in C57BL6/NJ mice.

Rank of differential expression transcriptome-wide, probe ID, $\mathrm{p}$-value, $\mathrm{t}$, a moderated $\mathrm{t}$ statistic, B, the log-odds of differential expression between the groups compared, fold change (FC) in mRNA expression level as compared to baseline, gene and gene name are listed in this chart. 


\begin{tabular}{|l|l|l|l|l|l|}
\hline Rank & ID & p-value & F & Gene & Gene name \\
\hline $\mathbf{1 5 8 3 5}$ & CfaAffx.18651.1.S1_at & $2.48 \mathrm{E}-02$ & 6.32 & IRF9 & $\begin{array}{l}\text { interferon } \\
\text { regulatory factor 9 }\end{array}$ \\
\hline $\mathbf{2 9 1 1 1}$ & CfaAffx.10779.1.S1_at & $2.14 \mathrm{E}-01$ & 1.91 & IRF7 & $\begin{array}{l}\text { interferon } \\
\text { regulatory factor 7 }\end{array}$ \\
\hline
\end{tabular}

Table 4: Statistically significant differential expression of IRF9 but not IRF7 in the ferret lung following infection with SARS-CoV-1.

Rank of differential expression transcriptome-wide, probe ID, p-value, F statistic, gene and gene name are listed in this chart. 
Table 5: Neither IRF9 nor IRF7 are differentially expressed in the ferret blood following infection with SARS-CoV-1.

Rank of differential expression transcriptome-wide, probe ID, $p$-value, $t$, a moderated tstatistic, B, the log-odds of differential expression between the groups compared, gene and gene name are listed in this chart.

PAGE 16 OF 20 


\begin{tabular}{|l|l|l|l|l|l|l|}
\hline Rank & ID & p-value & t & B & Gene & Gene name \\
\hline $\mathbf{3 6 1 1 6}$ & 48773 & 0.5145883 & 0.6898065 & -5.127 & IRF7 & $\begin{array}{l}\text { interferon } \\
\text { regulatory factor 7 }\end{array}$ \\
\hline $\mathbf{5 5 2 3 5}$ & 31099 & 0.8546024 & 0.1908025 & -5.314 & IRF9 & $\begin{array}{l}\text { interferon } \\
\text { regulatory factor 9 }\end{array}$ \\
\hline
\end{tabular}

Table 6: IRF7 and IRF9 are not differentially expressed in human cells following HCoV-229E infection in vitro.

Rank of differential expression transcriptome-wide, probe ID, $p$-value, $t$, a moderated tstatistic, B, the log-odds of differential expression between the groups compared, gene and gene name are listed in this chart. 


\section{Figure 1: Rapid transcriptional induction of IRF9 in human cells following MERS-} CoV London infection in vitro.

IRF9 mRNA expression levels are graphically depicted from 3 hours post-infection to 24 hours post-infection, with mean mRNA values marked and an exact $p$-value listed for each timepoint - the results of statistical tests evaluating significance of difference in IRF9 expression as compared to baseline (0 hours). 


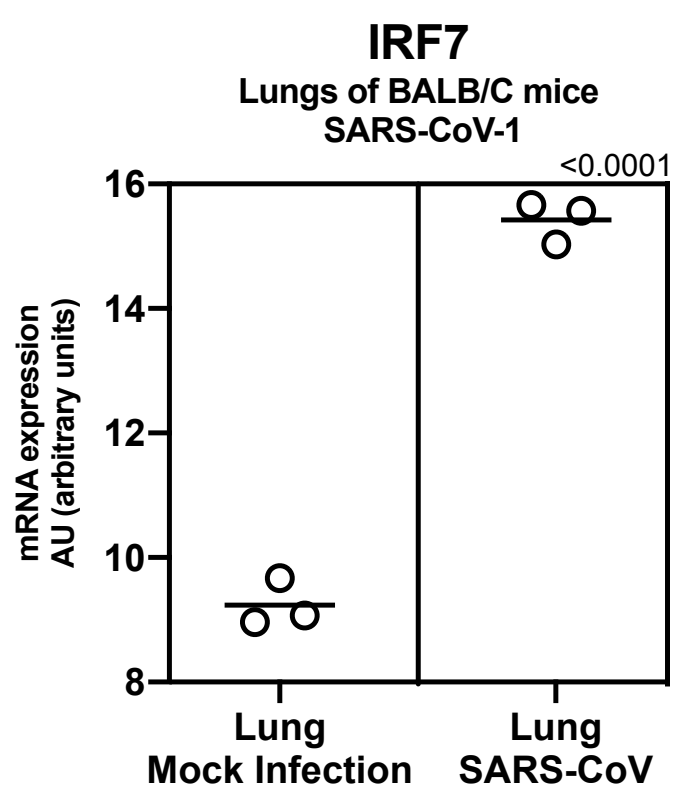

Figure 2: Transcriptional induction of IRF7 in the mouse lung in vivo following SARS-CoV-1 infection in BALB/c mice.

IRF7 mRNA expression levels are graphically depicted from in the mock-infected lung (lung) and in the mouse lung at 2 days post-infection with SARS-CoV-1, with mean mRNA values marked and an exact $p$-value listed, the result of a statistical test evaluating significance of difference in IRF7 expression as compared to baseline (mock infection). 


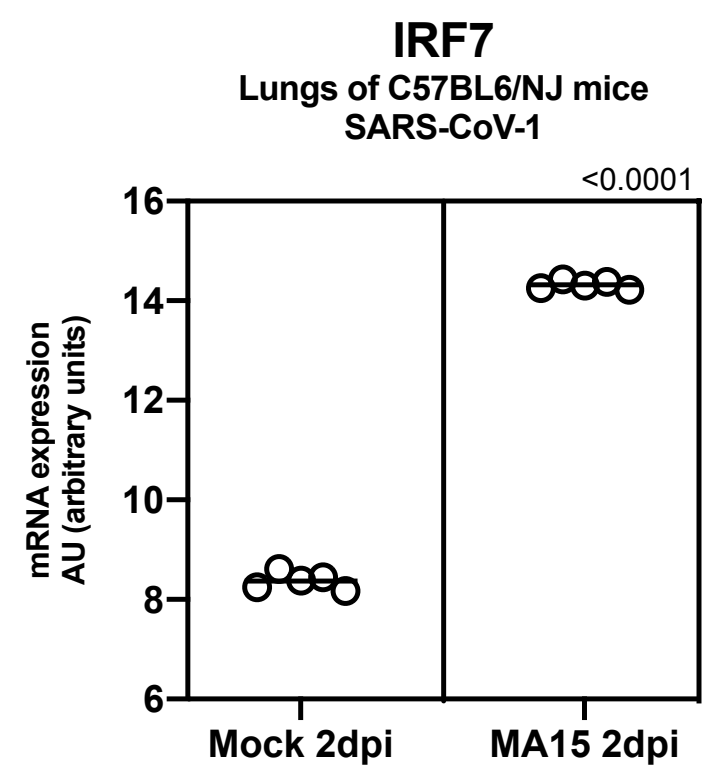

Figure 3: Transcriptional induction of IRF7 in the mouse lung in vivo following SARS-CoV-1 infection in C57BL6/NJ mice.

IRF7 mRNA expression levels are graphically depicted from in the mock-infected lung (lung) and in the mouse lung at 2 days post-infection with SARS-CoV-1, with mean mRNA values marked and an exact p-value listed, the result of a statistical test evaluating significance of difference in IRF7 expression as compared to baseline (mock infection). 